Research Article

\title{
Knockdown of MALAT1 Inhibits the Progression of Chronic Periodontitis via Targeting miR-769-5p/HIF3A Axis
}

\author{
Qinchao Chen, Meng Cao, and Hanyi Ge \\ Department of Stomatology, Zibo Central Hospital, No. 54, Gongqingtuan Road, Zhangdian District, \\ Zibo City Shandong Province 255000, China \\ Correspondence should be addressed to Hanyi Ge; gehanyi221@163.com
}

Received 17 September 2020; Revised 10 December 2020; Accepted 26 December 2020; Published 1 February 2021

Academic Editor: Yanming Xu

Copyright (C) 2021 Qinchao Chen et al. This is an open access article distributed under the Creative Commons Attribution License, which permits unrestricted use, distribution, and reproduction in any medium, provided the original work is properly cited.

\begin{abstract}
Purpose. Chronic periodontitis (CP) is a long-lasting inflammatory disease that seriously affects oral health. This study is aimed at investigating the regulatory mechanism of metastasis-associated lung adenocarcinoma transcript 1 (MALAT1) in CP. Methods. Primary human periodontal ligament cells (PDLCs) were treated with P. gingivalis lipopolysaccharide (LPS) to establish a CP model. Quantitative real-time PCR (qRT-PCR) was used to measure the expression of MALAT1 and miR-769-5p in gingival tissues of patients with CP and LPS-treated PDLCs. Cell viability was detected by 3-(4,5-dimethyl-2-thiazolyl)-2,5-diphenyl-2H-tetrazolium bromide (MTT) assay. Enzyme-linked immunosorbent assay (ELISA) was used to measure the levels of inflammatory cytokines. The protein levels of caspase-3, Bax, Bcl-2, and hypoxia-inducible factor (HIF) 3A were determined by western blot assay. Dual-luciferase reporter (DLR) assay was applied to validate the target relationships between miR-769-5p and MALAT1/HIF3A. Results. The expression of MALAT1 and HIF3A was enhanced, and the expression of miR-769-5p was reduced in gingival tissues of patients with CP and LPS-treated PDLCs. MALAT1 knockdown promoted cell viability and inhibited inflammation and cell apoptosis in LPS-treated PDLCs. MALAT1 targeted miR-769-5p and negatively regulated miR769-5p expression. miR-769-5p overexpression promoted cell viability and inhibited inflammation and cell apoptosis in LPStreated PDLCs. Besides, miR-769-5p targeted HIF3A and negatively modulated HIF3A expression. Both miR-769-5p inhibition and HIF3A overexpression reversed the inhibitory effects of MALAT1 silencing on LPS-induced PDLC injury in vitro. Conclusion. MALAT1 knockdown attenuated LPS-induced PDLC injury via regulating the miR-769-5p/HIF3A axis, which may supply a new target for CP treatment.
\end{abstract}

\section{Introduction}

Periodontitis is a common inflammatory disease, which is caused by the imbalance of periodontal microbiota, such as Porphyromonas gingivalis (P. gingivalis) [1]. Chronic periodontitis $(\mathrm{CP})$ is a long-lasting periodontitis disease and a chronic noncommunicable disease that destroys the integrity of the periodontium and leads to gingival swelling and bleeding, bone loss, and tooth exfoliation [2]. According to data from the World Health Organization, CP is one of the chronic noncommunicable diseases that seriously affect people's quality of life $[3,4]$. The current treatment methods of periodontitis including scaling, surgery, and systemic antibiotics have made great progress, but the treatment effect remains dissatisfying [5]. Thus, it is necessary to understand the molecular mechanism of $\mathrm{CP}$ to improve its therapy.

Long noncoding RNAs (lncRNAs) above $200 \mathrm{nt}$ have been recognized to be involved in many human diseases [6]. Recently, many researches showed that lncRNAs are aberrantly expressed in periodontitis and therefore play essential roles in the development of periodontitis [7, 8]. LINC00687 expression is upregulated, whereas the expression of LBX2AS1 and LINC01566 is downregulated in periodontitis samples [9]. IncRNA PTCSC3 expression is decreased in periodontal ligament stem cells (PDLSCs) of CP patients, and its overexpression inhibits PDLSC proliferation [10]. Notably, 
metastasis-associated lung adenocarcinoma transcript 1 (MALAT1) is also related to periodontitis progression [11]. MALAT1 expression is obviously increased in PDLSCs isolated from periodontitis patients, and its overexpression promotes cell proliferation [12]. MALAT1 is highly expressed in inflammatory gingival tissues of $\mathrm{CP}$ and promotes inflammatory cytokine secretion in human gingival fibroblast (HGF) cells [13]. However, the detailed molecular mechanism of MALAT1 in CP needs further study.

MicroRNAs (miRNAs) are a type of noncoding RNAs that are implicated in some pathogenic events, including periodontitis [14]. Previous studies have revealed that some miRNAs affect the occurrence and development of periodontitis, such as miR-21 [15], miR-182 [16], and miR-155-5p [17]. Additionally, miR-769-5p acts as a molecular biomarker and is involved in some immunological disorders. Chen et al. have discovered that miR-769-5p expression is upregulated in rheumatoid arthritis (RA) and gouty arthritis (GA) [18]. Besides, miR-769-5p expression is decreased in lipopolysaccharide- (LPS-) treated periodontal ligament cells (PDLCs) [19]. Previous studies suggested that the roles of miRNAs in periodontitis are regulated by $\operatorname{lncRNAs}$. For example, lncRNA TUG1 mitigates cell injury and cytokine production by regulating miR-498 in LPS-treated PDLCs [20]. The inhibition effects of miR-20a on secretion of inflammatory cytokines are regulated by MALAT1 in LPStreated HGF cells [13]. However, the exact role of miR-769$5 \mathrm{p}$ and the interaction with MALAT1 in CP are unrevealed.

Generally, hypoxia-inducible factor (HIF) 3A is known as an oncogene in several types of human cancers, such as in ovarian [21], prostate [22], breast [23], pancreatic [24], and non-small-cell lung [25] cancers. In addition, two members (HIF1A and HIF2A) of the HIF family are confirmed to upregulate in aging gingival tissues with hypoxic stress [26]. Notably, a recent study conducted by Jia et al. has demonstrated that HIF3A expression is increased in CP tissues and LPS-induced PDLCs and HIF3A overexpression partly reversed the effects of miR-210 upregulation on cell viability, apoptosis, and inflammation factor expression in LPS-treated PDLCs [27]. Nevertheless, whether HIF3A interacts with the MALAT1/miR-769-5p axis in participation of CP progression is relatively unknown.

In our study, the expression of MALAT1, miR-769-5p, and HIF3A was measured in gingival tissues of patients with CP and LPS-treated PDLCs. Then, we investigated the effects of MALAT1 knockdown or miR-769-5p overexpression on cell viability, inflammation, and cell apoptosis in LPStreated PDLCs. Furthermore, we further explored the regulatory mechanism of MALAT1/miR-769-5p/HIF3A axis on LPS-induced PDLC injury. This study may offer an underlying target for improving treatment strategy of CP.

\section{Material and Methods}

2.1. Gingival Sample Collection. A total of 26 patients ( 12 males, 14 females, median age: $36 \pm 11$ years old) with $\mathrm{CP}$ and 17 healthy controls ( 8 males, 9 females, median age: 32 \pm 8 years old) from March 2018 to September 2019 were chosen in our hospital. The gingival tissue samples from CP patients were obtained during surgical therapy [28], and healthy gingival tissue samples from healthy controls were collected during crown-lengthening procedures. This study obtained the ratification of the local ethics committee, and written informed consents were acquired from all individuals.

2.2. Cell Culture and Treatment. In line with previously described methods, PDLCs were isolated from healthy periodontal ligament in the middle third of the periodontal ligament root of the third molars of 5 healthy volunteers [29]. The cells were cultured in Dulbecco's Modification of Eagle's Medium (DMEM) (Gibco, Carlsbad, CA, USA) containing $10 \%$ fetal bovine serum (FBS, Gibco), $100 \mathrm{U} / \mathrm{mL}$ penicillin, and $100 \mu \mathrm{g} / \mathrm{mL}$ streptomycin at $37^{\circ} \mathrm{C}$ in an incubator with $5 \%$ $\mathrm{CO}_{2}$. PDLCs in the third generation were used in the next experiments. To establish a CP model, PDLCs were treated with $100 \mathrm{ng} / \mathrm{mL} P$. gingivalis LPS (Sigma, St Louis, MI, USA) for $72 \mathrm{~h}$.

2.3. Cell Transfection. Small interfering RNA negative control (si-NC), si-MALAT1, miRNA negative control (miR-NC), miR-769-5p mimics, miR-769-5p inhibitor, and pcDNAHIF3A were acquired from GenePharma (Shanghai, China). PDLCs were planted into 6-well plates and grew to $80 \%$ confluence. The above factors were transfected into PDLCs utilizing Lipofectamine 3000 (Invitrogen, Carlsbad, CA, USA) in accordance with the manufacturer's instructions. The cells were collected at $48 \mathrm{~h}$ after transfection.

2.4. Quantitative Real-Time Polymerase Chain Reaction ( $q R T-P C R)$. Total RNA from gingival tissues and PDLCs was obtained by TRIzol (Invitrogen), and cDNA was produced by utilizing the PrimeScript RT Master Mix (for gene amplification, Takara) or Mir-X miRNA First-Strand Synthesis Kit (for miRNA amplification, Takara). The qRTPCR was performed by a SYBR Green PCR Kit (Takara). Primer sequences are enumerated in Table 1. The qRTPCR conditions were as follows: $94^{\circ} \mathrm{C}$ for $10 \mathrm{~min}$, followed by 40 cycles at $94^{\circ} \mathrm{C}$ for $15 \mathrm{~s}, 60^{\circ} \mathrm{C}$ for $1 \mathrm{~min}$, and $72^{\circ} \mathrm{C}$ for $1 \mathrm{~min}$. The expression of MALAT1 and HIF3A was normalized by GAPDH. U6 acted as the endogenous control for miR-769-5p. Relative gene expression was measured by $2^{-\Delta \Delta \mathrm{Ct}}$ method.

2.5. Western Blot. Total proteins were extracted from PDLCs using RIPA buffer (Beyotime, Shanghai, China). Equal protein samples were separated by $10 \%$ sodium dodecyl sulfate polyacrylamide gel electrophoresis (SDS-PAGE) and then transferred to PVDF membranes (Bio-Rad, Inc., Hercules, CA, USA). Then, the membranes were blocked with $5 \%$ nonfat milk for $1 \mathrm{~h}$ and incubated overnight at $4^{\circ} \mathrm{C}$ with primary antibodies of $\operatorname{Bax}(1: 1,000$, ab32503, Abcam, Cambridge, UK), Bcl-2 (1:1,000, ab32124, Abcam), HIF3A (1:1,000, ab10134, Abcam), caspase-3 (1:1,000, ab32351, Abcam), and $\beta$-actin ( $1: 1,000$, ab5694, Abcam). After the membranes were washed with tris-buffered-saline Tween (TBST), a secondary antibody (1:5,000, ab6728, Abcam) was added to incubate with the membranes at $37^{\circ} \mathrm{C}$ for $2 \mathrm{~h}$. The immunoblots were quantified by using ImageLab software (Bio-Rad, Inc., Hercules, CA, USA). The relative protein levels of $\mathrm{Bax}, \mathrm{Bcl}-2, \mathrm{HIF} 3 \mathrm{~A}$, and caspase- 3 were normalized by $\beta$-actin. 
TABLE 1: Primers for quantitative real-time polymerase chain reaction (qRT-PCR).

\begin{tabular}{lc}
\hline Gene & ${\text { Sequences }\left(5^{\prime}-3^{\prime}\right)}^{\text {MALAT1-F }}$ \\
MALAT1-R & AAAGCAAGGTCTCCCCACAAG \\
miR-769-5p-F & GGTCTGTGCTAGATCAAAAGGCA \\
miR-769-5p-R & ACACTCCAGCTGGGTGAGACCTCTGGGTTCTG \\
HIF3A-F & CTCAACTGGTGTCGTGGA \\
HIF3A-R & CTTTCTGCTCTTTCCTCTCAGC \\
GAPDH-F & GCTCATTCAGGTTCAGGAGTG \\
GAPDH-R & GCGAGATCGCACTCATCATCT \\
U6-F & TCAGTGGTGGACCTGACC \\
U6-R & CTCGCTTCGGCAGCACA \\
\hline
\end{tabular}

2.6. MTT Assay. Following transfection and LPS treatment, PDLCs were planted into 96 -well plates $\left(3 \times 10^{4}\right.$ cells/well $)$. The MTT ( $5 \mathrm{mg} / \mathrm{mL}$, Sigma) was added into each well for $4 \mathrm{~h}$; then, DMSO (200 $\mu \mathrm{L}$, Sigma) solution was subjoined to dissolve formazan crystal. Finally, the absorbance at $570 \mathrm{~nm}$ was detected by a microplate reader (Molecular Devices; Hercules, CA, USA).

2.7. Enzyme-Linked Immunosorbent Assay (ELISA). The supernatants of PDLCs with transfection and LPS treatment were gathered. ELISA kits (Boster, Wuhan, China) were used to measure the levels of interleukin- (IL-) $1 \beta$, IL-6, and tumor necrosis factor- (TNF-) $\alpha$ in supernatants according to the manufacturer's protocol. The absorbance at $450 \mathrm{~nm}$ was read using a microplate reader (Molecular Devices).

2.8. Target Prediction. The miRNA targets of MALAT1 were predicted using StarBase software (http://starbase.sysu.edu $. \mathrm{cn} /$ ), and 357 miRNA targets were predicted. Among these miRNA targets, miR-769-5p was selected due to its important role in LPS-induced periodontal ligament cells [19]. In addition, the regulatory relationship between MALAT1 and miR-769-5p has not been studied yet. The mRNA targets of miR-769-5p were predicted using StarBase software and TargetScan software (http://www.targetscan.org/vert_72/). A total of 2,321 and 3,669 targets were predicted, respectively. Afterwards, HIF3A was chosen due to its crucial role in periodontitis [27] and unknown relationship with miR-769-5p.

2.9. Dual-Luciferase Reporter (DLR) Assay. The fragments of MALAT1/HIF3A containing the wild-type or mutant binding sites of miR-769-5p were cloned into the pGL3 vector (Promega, Madison, WI, USA) to produce wild-type luciferase reporter vectors (MALAT1-wt, HIF3A-wt) or mutant luciferase reporter vectors (MALAT1-mut, HIF3A-mut), respectively. The above vectors and miR-NC/miR-769-5p mimics were transfected into PDLCs by Lipofectamine 3000 (Invitrogen). After transfection of $48 \mathrm{~h}$, a dualluciferase reporter assay kit (Promega) was used to detect the luciferase activities.
2.10. Statistical Analysis. All data were evaluated by applying SPSS 22.0 software (IBM Corp., Armonk, NY, USA) and presented as the mean \pm standard deviation. The comparisons between two groups or among multiple groups were performed by Student's $t$-test or one-way ANOVA followed by Tukey's post hoc test. A value of $P<0.05$ was deemed as a significant difference.

\section{Results}

3.1. Inhibition of MALAT1 Attenuates LPS-Induced PDLC Injury. MALAT1 expression was increased in gingival tissues of patients with CP compared with gingival tissues of healthy controls $(P<0.01$, Figure 1(a)). As illustrated in Figure 1(b), MALAT1 expression in LPS-treated PDLCs was higher than that in control PDLCs $(P<0.01)$. To explore the role of MALAT1 in CP, PDLCs were transfected with si-NC or siMALAT1. The results displayed that MALAT1 knockdown suppressed MALAT1 expression in PDLCs $(P<0.01$, Figure $1(\mathrm{c})$ ). MTT assay indicated that cell viability was inhibited in LPS-treated PDLCs compared with control PDLCs, while silencing of MALAT1 enhanced cell viability in LPStreated PDLCs $(P<0.01$, Figure $1(\mathrm{~d}))$. Moreover, the levels of IL-6, IL- $1 \beta$, and TNF- $\alpha$ were obviously increased in LPStreated PDLCs compared with control PDLCs, while knockdown of MALAT1 inhibited the release of inflammatory cytokines in LPS-treated PDLCs $(P<0.01$, Figures $1(\mathrm{e})-$ $1(\mathrm{~g})$ ). Further studies indicated that the protein levels of Bax and caspase- 3 were enhanced, whereas Bcl-2 expression was reduced in LPS-treated PDLCs compared with control PDLCs $(P<0.01$, Figures $1(\mathrm{~h})$ and $1(\mathrm{i}))$. Silencing of MALAT1 reduced the Bax and caspase- 3 protein levels and enhanced Bcl-2 expression in LPS-treated PDLCs $(P<0.01$, Figures $1(\mathrm{~h})$ and 1(i)). These data revealed that silencing of MALAT1 enhanced cell viability and inhibited inflammation and apoptosis in LPS-treated PDLCs.

3.2. miR-769-5p Is a Target of MALAT1. The underlying target site between MALAT1 and miR-769-5p was predicted using StarBase software (Figure 2(a)). To investigate the relationship between MALAT1 and miR-769-5p, si-NC or si-MALAT1 was transfected into PDLCs. The results 


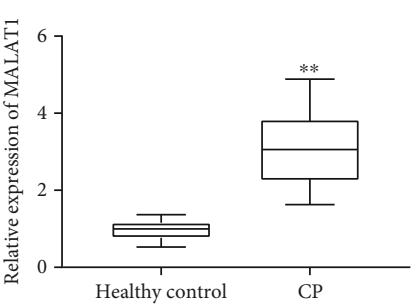

(a)

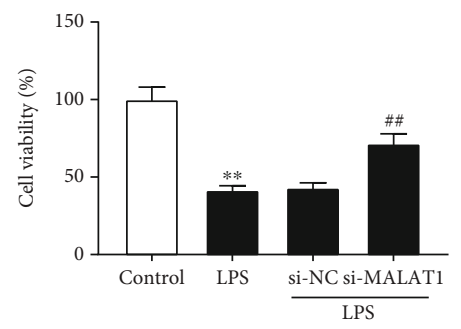

(d)

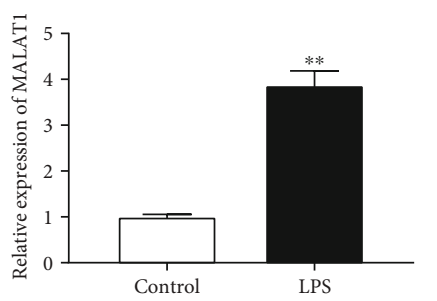

(b)

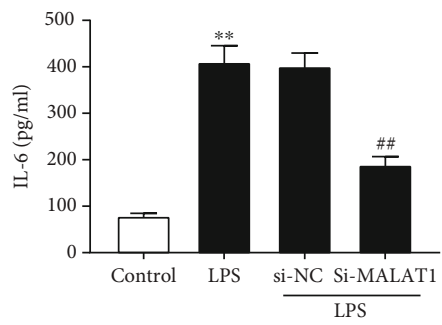

(e)

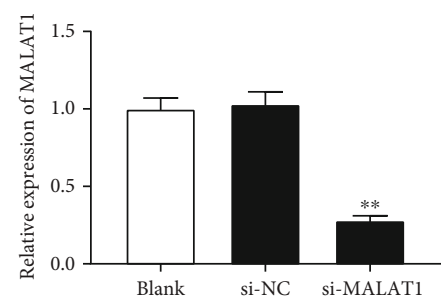

(c)

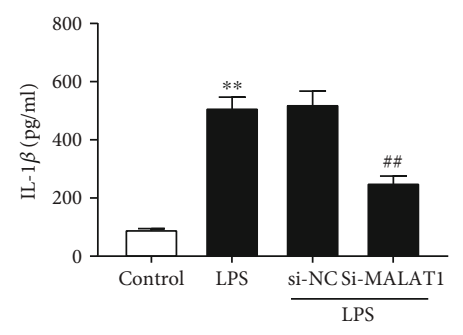

(f)

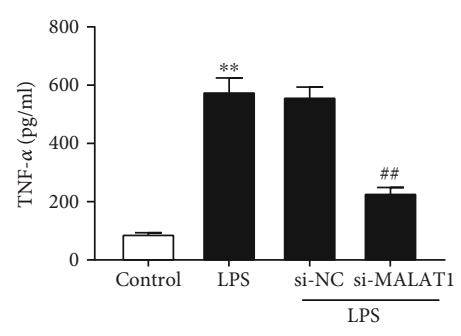

(g)

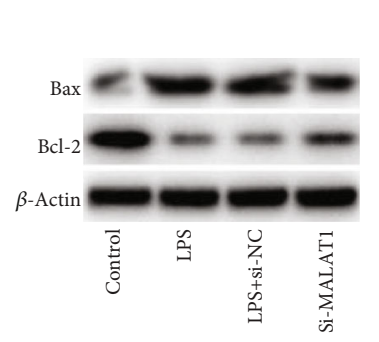

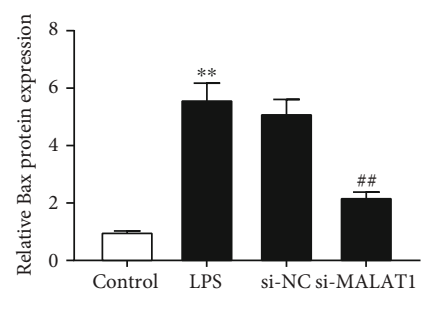

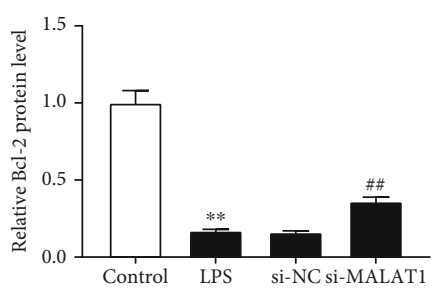

(h)
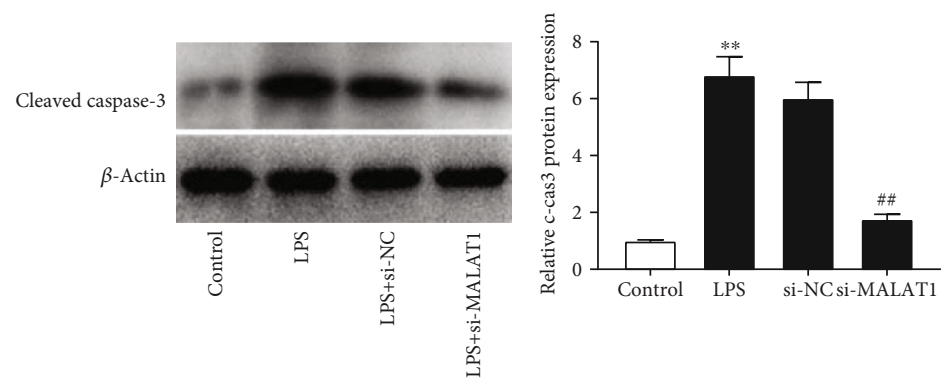

(i)

Figure 1: Inhibition of metastasis-associated lung adenocarcinoma transcript 1 (MALAT1) attenuated lipopolysaccharide- (LPS-) induced periodontal ligament cell (PDLC) injury. (a) The expression of MALAT1 was detected by quantitative real-time polymerase chain reaction (qRT-PCR) in gingival tissues of patients with chronic periodontitis $(\mathrm{CP})$ and healthy controls. ${ }^{* *} P<0.01$ vs. healthy controls. (b) The expression of MALAT1 was detected by qRT-PCR in LPS-treated PDLCs and control cells. ${ }^{* *} P<0.01$ vs. control cells. (c) The expression of MALAT1 was detected by qRT-PCR in PDLCs transfected with si-NC or si-MALAT1. ${ }^{* *} P<0.01$ vs. si-NC. (d) Cell viability was detected by MTT assay in LPS-treated PDLCs transfected with si-NC or si-MALAT1. ${ }^{* *} P<0.01$ vs. control; ${ }^{\# \#} P<0.01$ vs. si-NC. (e-g) The concentrations of interleukin- (IL-) 6 , IL- $1 \beta$, and tumor necrosis factor- $\alpha$ (TNF- $\alpha$ ) were measured by enzyme-linked immunosorbent assay (ELISA) in supernatants of LPS-treated PDLCs transfected with si-NC or si-MALAT1. ${ }^{* *} P<0.01$ vs. control; ${ }^{\# \#} P<0.01$ vs. si-NC. (h) The protein levels of Bax and Bcl-2 were detected by western blot in LPS-treated PDLCs transfected with si-NC or si-MALAT1. ${ }^{* *} P<$ 0.01 vs. control; ${ }^{\#} P<0.01$ vs. si-NC. (i) The protein level of caspase-3 was detected by western blot in LPS-treated PDLCs transfected with si-NC or si-MALAT1. ${ }^{* *} P<0.01$ vs. control; ${ }^{\# \#} P<0.01$ vs. si-NC.

uncovered that miR-769-5p expression in the si-MALAT1 group was increased in comparison with that in the si-NC group $(P<0.01$, Figure $2(\mathrm{~b}))$. Moreover, DLR assay showed that the luciferase activity of MALAT1-wt in the miR-769$5 \mathrm{p}$ mimics group was decreased as compared to that in the miR-NC group $(P<0.01$, Figure $2(\mathrm{c}))$. These results suggested that miR-769-5p was a downstream target of MALAT1.
3.3. miR-769-5p Overexpression Alleviates LPS-Induced PDLC Injury. miR-769-5p expression was reduced in gingival tissues of patients with CP compared with gingival tissues of healthy controls $(P<0.01$, Figure $3(\mathrm{a}))$. At the same time, miR-769-5p expression was decreased in LPS-treated PDLCs as compared to control PDLCs $(P<0.01$, Figure $3(\mathrm{~b}))$. To explore the role of miR-769-5p in CP, miR-NC, miR-769$5 \mathrm{p}$ mimics, or miR-769-5p inhibitor was transfected into 


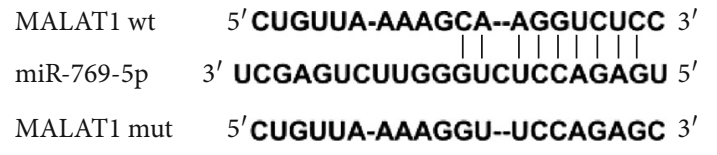

(a)

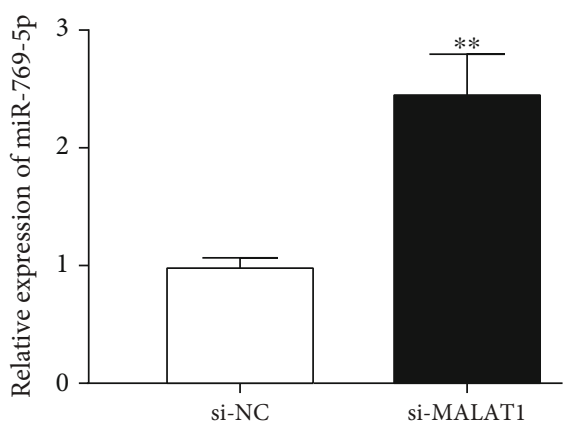

(b)

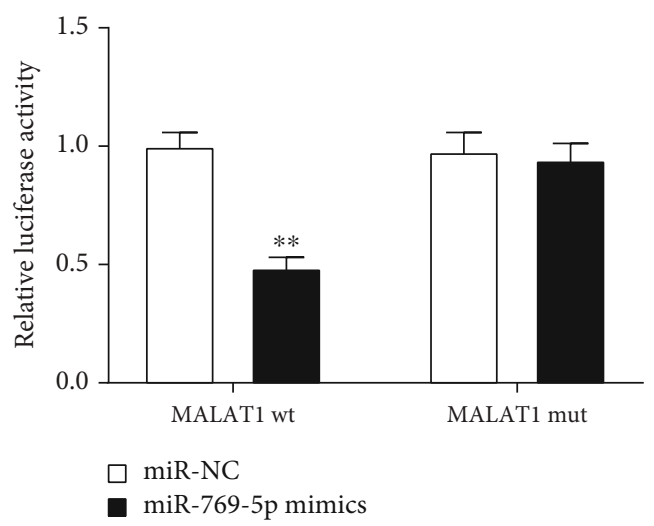

(c)

Figure 2: miR-769-5p was a target of MALAT1. (a) The target sites between MALAT1 and miR-769-5p were predicted by StarBase. (b) The expression of miR-769-5p was measured by qRT-PCR in PDLCs transfected with si-NC or si-MALAT1. ${ }^{* *} P<0.01$ vs. si-NC. (c) Dualluciferase reporter (DLR) assay confirmed the relationship between MALAT1 and miR-769-5p in PDLCs. ${ }^{* *} P<0.01$ vs. miR-NC.

PDLCs. We discovered that miR-769-5p mimics enhanced miR-769-5p expression in PDLCs, while miR-769-5p inhibitor caused a reverse effect $(P<0.01$, Figure 3(c)). MTT assay demonstrated that the cell viability was enhanced in the miR769-5p mimics group compared to the miR-NC group $(P<0.01$, Figure $3(\mathrm{~d}))$. The levels of IL-6, IL-1 $\beta$, and TNF$\alpha$ were obviously decreased in the miR-769-5p mimics group as compared to the miR-NC group in LPS-treated PDLCs $(P<0.01$, Figures 3(e)-3(g)). Western blot assay showed that miR-769-5p mimics reduced the protein levels of Bax and caspase-3 and enhanced Bcl-2 expression in LPS-treated PDLCs $(P<0.01$, Figures $3(\mathrm{~h})$ and 3(i)). These data uncovered that overexpression of miR-769-5p enhanced cell viability and inhibited inflammation and apoptosis in LPS-treated PDLCs.

3.4. HIF3A Is a Target of miR-769-5p. The potential target site between miR-769-5p and HIF3A was predicted by StarBase and TargetScan software (Figure 4(a)). To explore the relationship between miR-769-5p and HIF3A, miR-NC or miR-769-5p mimics was transfected into PDLCs. We found that the expression of HIF3A was obviously decreased in the miR-769-5p mimics group compared with the miR-NC group $(P<0.01$, Figure $4(\mathrm{~b}))$. Moreover, DLR assay displayed that miR-769-5p mimics inhibited the luciferase activity of HIF3A-wt in PDLCs $(P<0.01$, Figure $4(\mathrm{c}))$. The above results implied that HIF3A was a direct target gene of miR-769-5p.
3.5. MALAT1 Knockdown Inhibits LPS-Induced PDLC Injury through Regulating miR-769-5p/HIF3A Axis. HIF3A expression was increased in gingival tissues of patients with $\mathrm{CP}$ as compared to gingival tissues of healthy control $(P<0.01$, Figure 5(a)). The relative protein level of HIF3A was increased in LPS-treated PDLCs compared with control PDLCs $(P<0.01$, Figure 5(b)). Moreover, MALAT1 knockdown inhibited HIF3A expression in LPS-treated PDLCs, while miR-769-5p inhibition reversed this inhibitory effect $(P<0.01$, Figure 5(c)). Then, the joint effects of silencing of MALAT1 and miR-769-5p knockdown or HIF3A overexpression on LPS-induced PDLC injury were studied. We discovered that miR-769-5p downregulation or HIF3A overexpression partly inhibited the promoting effect of MALAT1 silencing on viability of LPS-treated PDLCs $(P<0.01$, Figure 5(d)). Besides, both miR-769-5p inhibition and HIF3A overexpression reversed the inhibitory effects of si-MALAT1 on the secretion of IL-6, IL-1 $\beta$, and TNF$\alpha$ in LPS-treated PDLCs $(P<0.01$, Figures 5(e)-5(g)). Further studies showed that both the low expression of miR-769-5p and high expression of HIF3A reversed the inhibiting effects of MALAT1 knockdown on the levels of Bax and caspase-3 and the promoting effect on Bcl-2 protein level $(P<0.01$, Figures $5(\mathrm{~h})$ and $5(\mathrm{i}))$. The results suggested that MALAT1 knockdown inhibited LPSinduced PDLC injury through regulating miR-769-5p and HIF3A expression. 


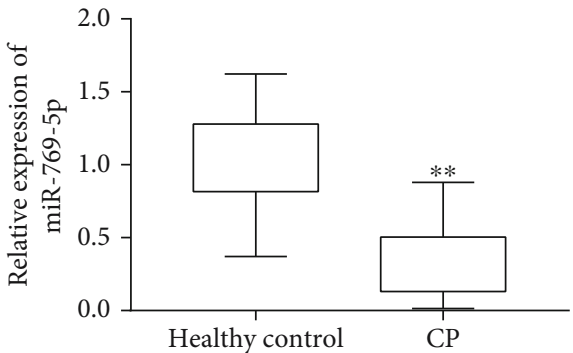

(a)

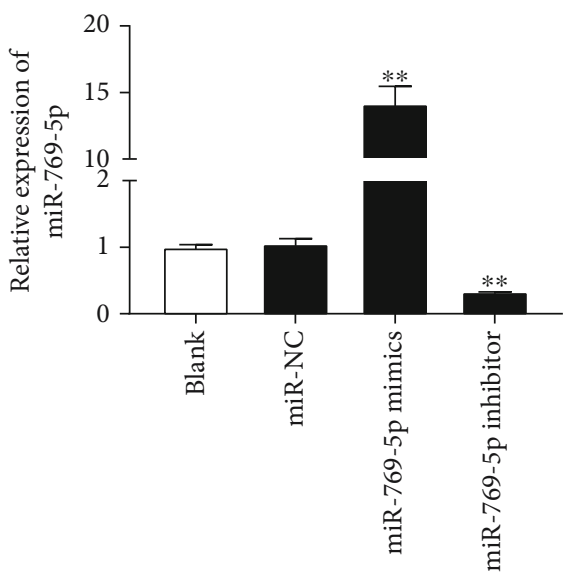

(c)

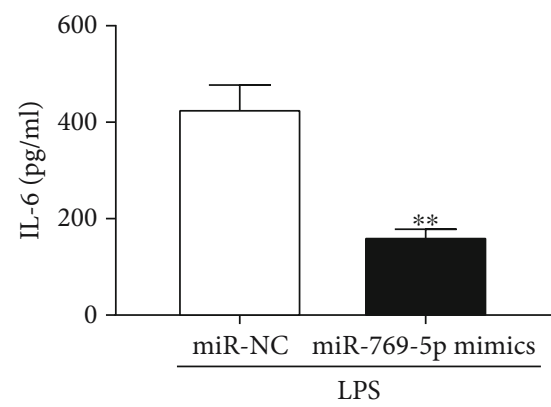

(e)



(b)

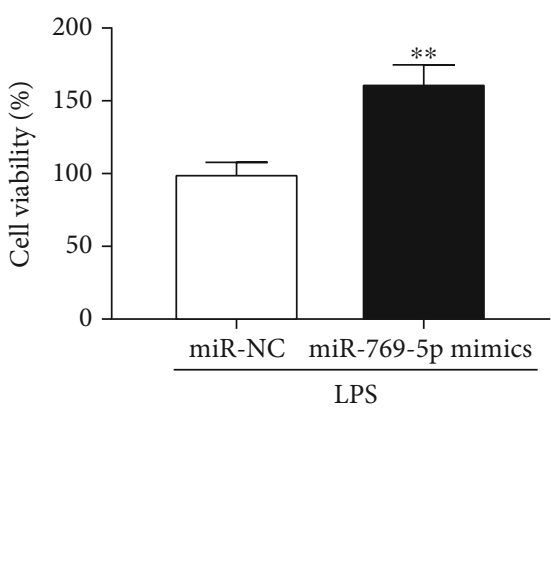

(d)

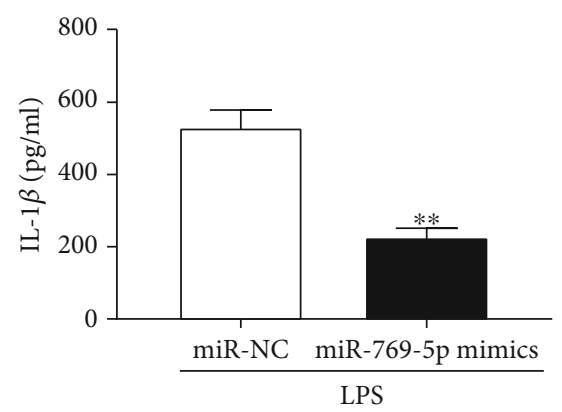

(f)

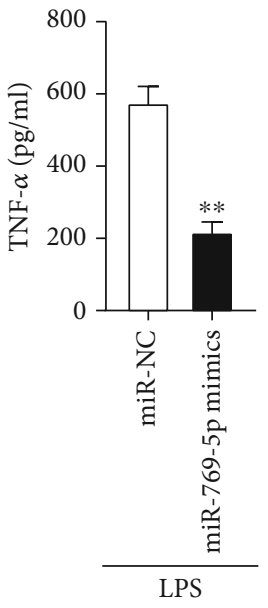

(g)

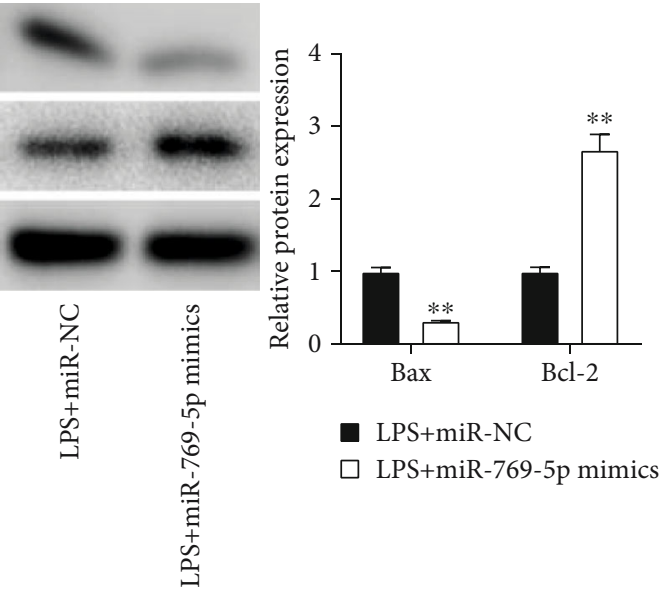

(h)

Figure 3: Continued. 


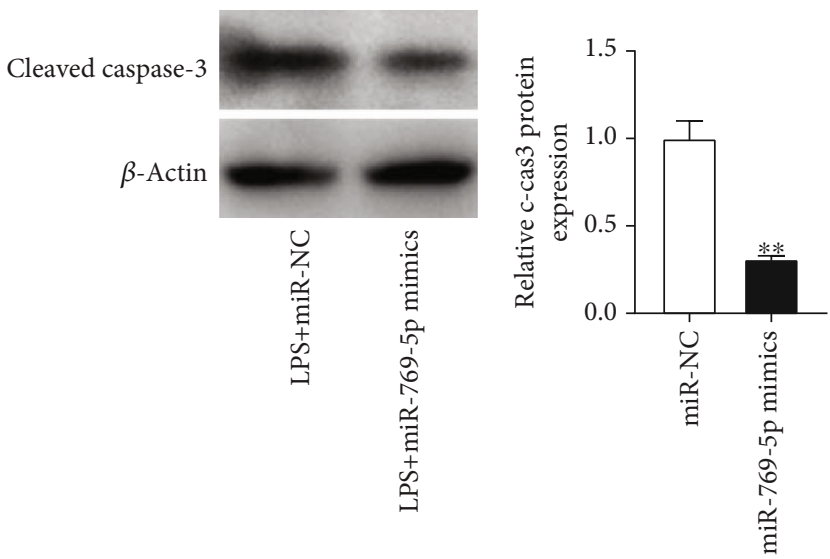

(i)

FIGURE 3: miR-769-5p mimics alleviated LPS-induced PDLC injury. (a) The expression of miR-769-5p was detected by qRT-PCR in gingival tissues of patients with CP and healthy controls. ${ }^{* *} P<0.01$ vs. healthy controls. (b) The expression of miR-769-5p was detected by qRT-PCR in LPS-treated PDLCs and control cells. ${ }^{* *} P<0.01$ vs. control cells. (c) The expression of miR-769-5p was detected by qRT-PCR in PDLCs transfected with miR-NC, miR-769-5p mimics, or miR-769-5p inhibitor. ${ }^{* *} P<0.01$ vs. the miR-NC group. (d) Cell viability was detected by MTT assay in LPS-treated PDLCs transfected with miR-NC or miR-769-5p mimics. ${ }^{* *} P<0.01$ vs. the miR-NC group. (e-g) The concentrations of IL-6, IL- $1 \beta$, and TNF- $\alpha$ were measured by ELISA in supernatants of LPS-treated PDLCs transfected with miR-NC or miR-769-5p mimics. ${ }^{* *} P<0.01$ vs. the miR-NC group. (h) The protein levels of Bax and Bcl-2 were detected by western blot in LPStreated PDLCs transfected with miR-NC or miR-769-5p mimics. ${ }^{* *} P<0.01$ vs. the miR-NC group. (i) The protein level of caspase-3 was detected by western blot in LPS-treated PDLCs transfected with miR-NC or miR-769-5p mimics. ${ }^{* *} P<0.01$ vs. the miR-NC group.

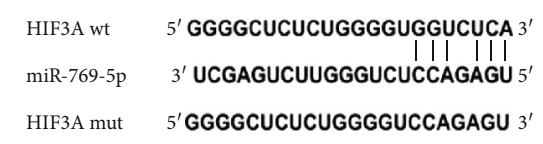

(a)
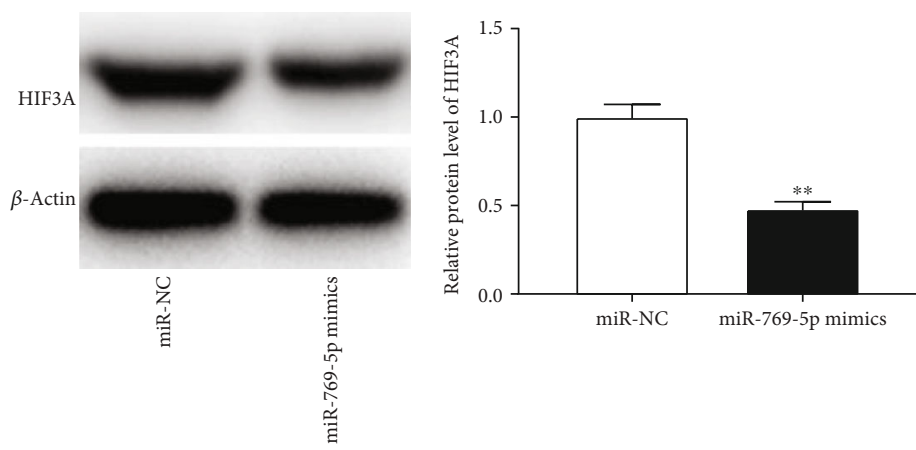

(b)

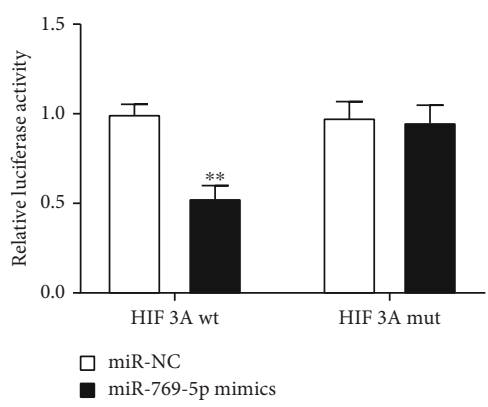

(c)

Figure 4: HIF3A was a target of miR-769-5p. (a) The target sites between miR-769-5p and HIF3A were predicted by StarBase. (b) The expression of HIF3A was detected by western blot in PDLCs transfected with miR-NC or miR-769-5p mimics. ${ }^{* *} P<0.01$ vs. miR-NC. (c) DLR assay confirmed the relationship between miR-769-5p and HIF3A in PDLCs. ${ }^{* *} P<0.01$ vs. miR-NC. 


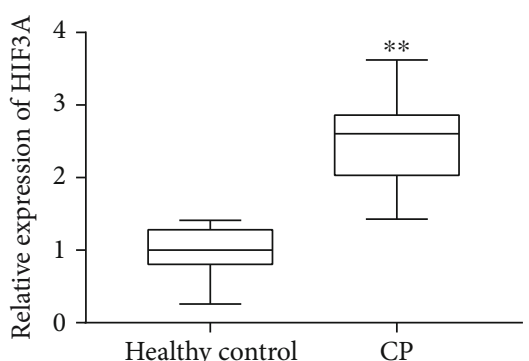

(a)

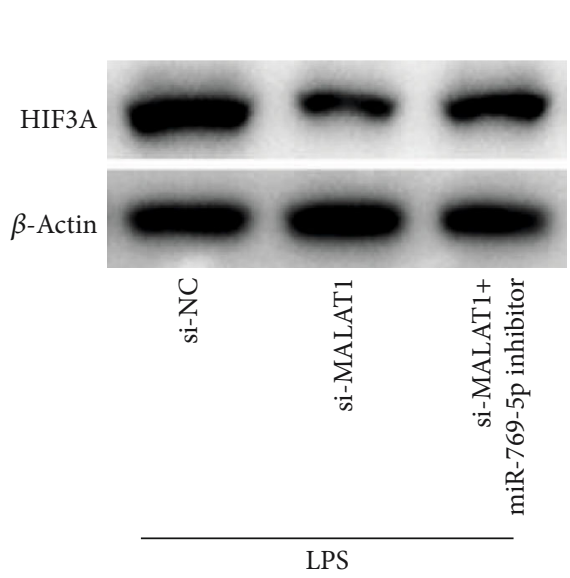

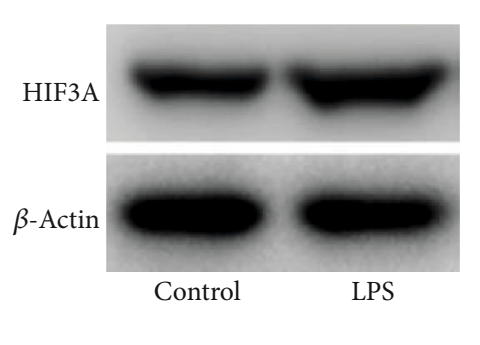

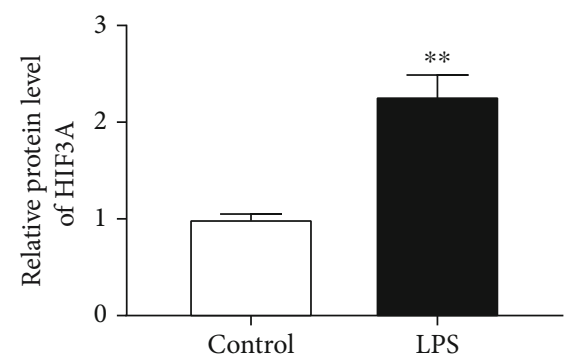

(b)
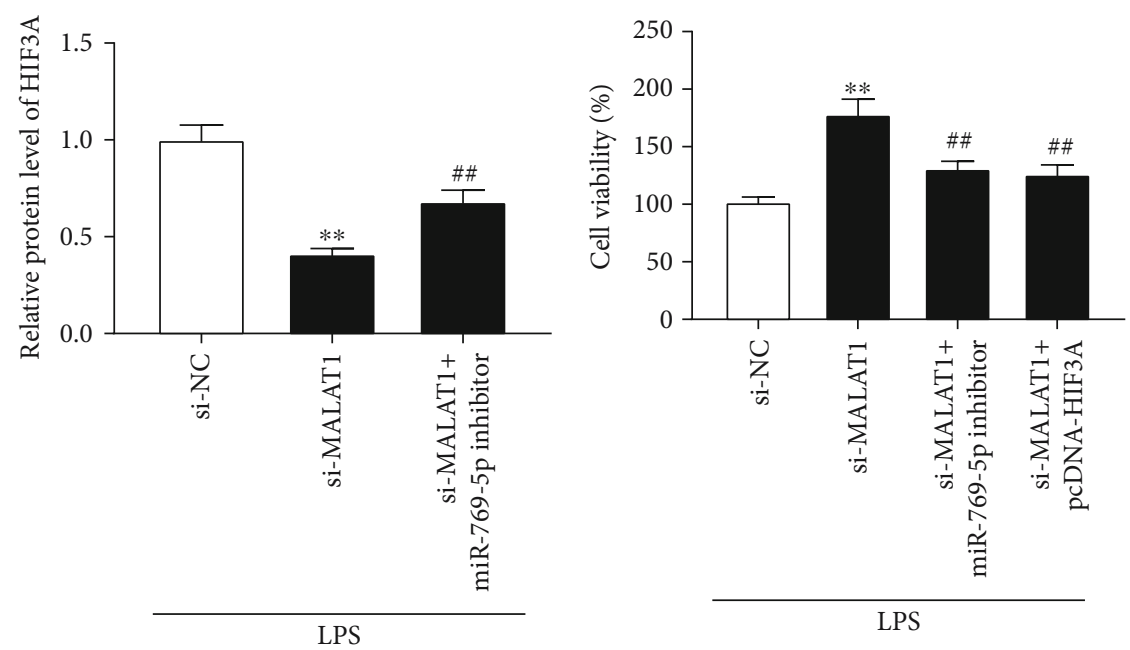

(c)

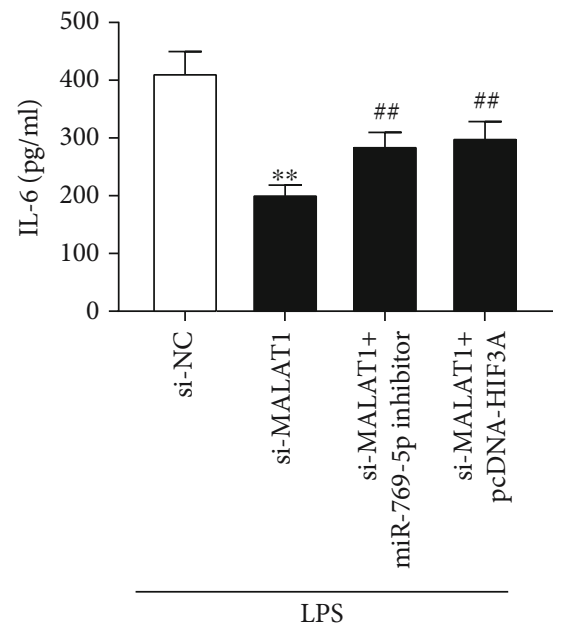

(e)

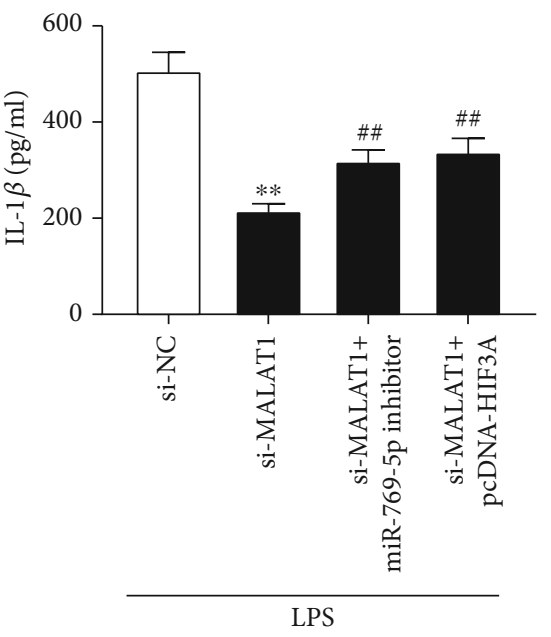

(f)

Figure 5: Continued. 

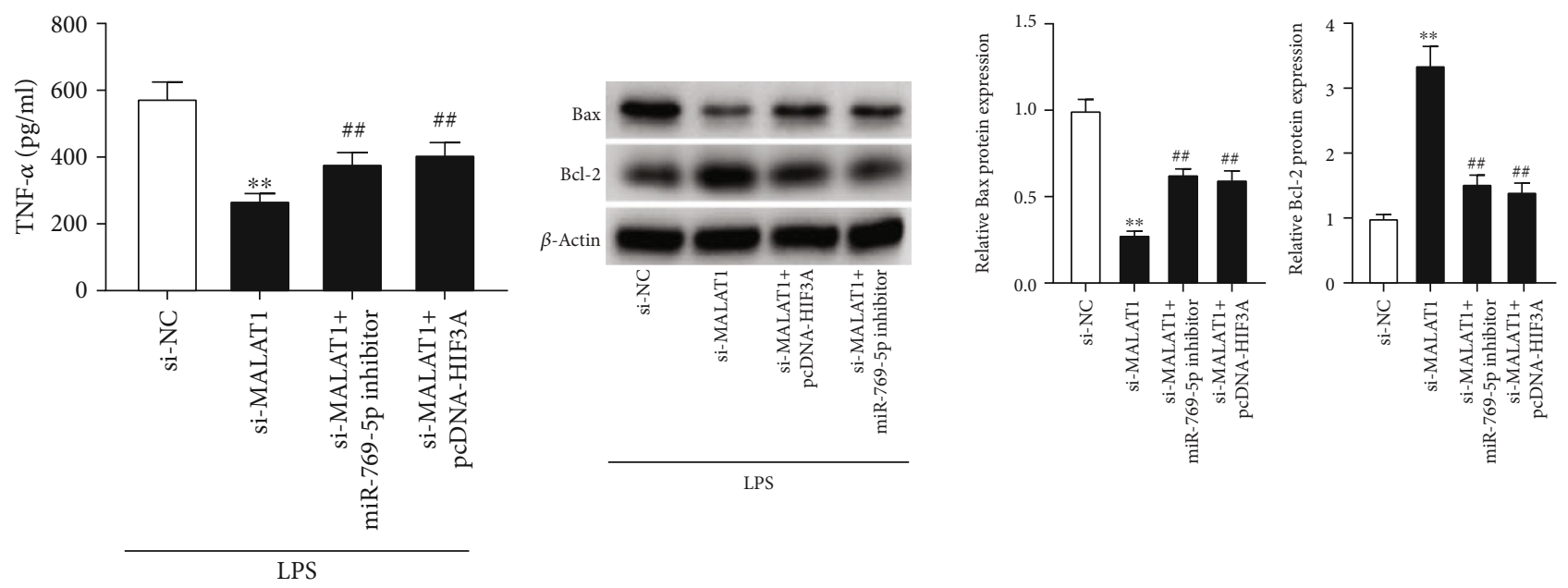

(g)

(h)
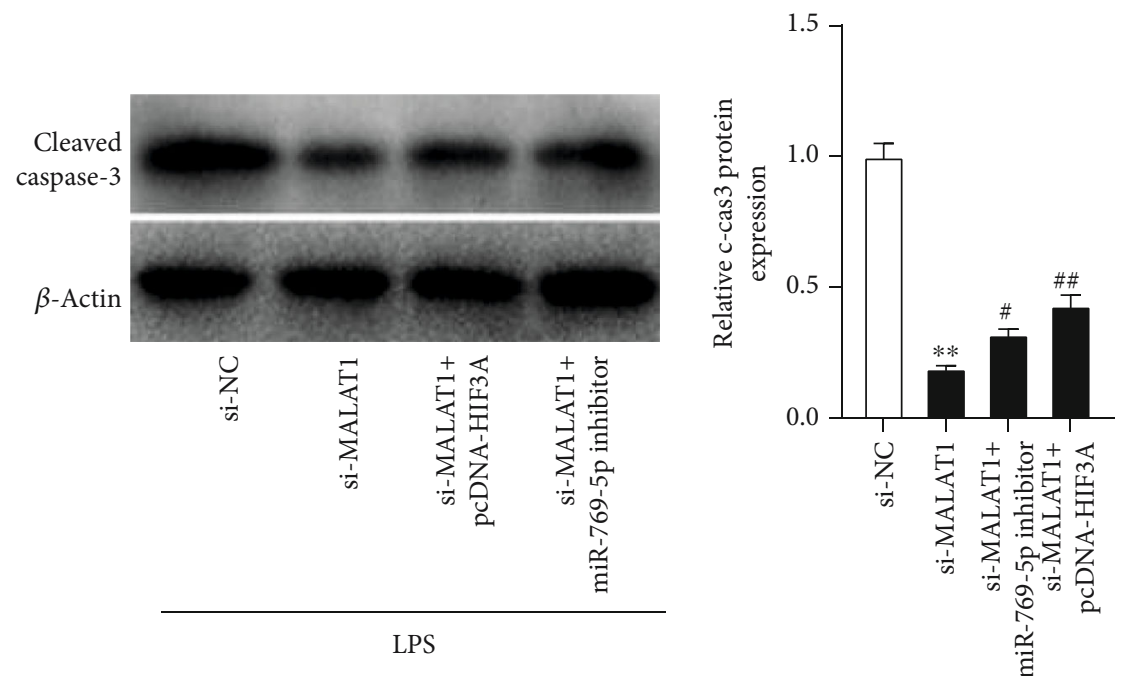

(i)

Figure 5: MALAT1 knockdown inhibited LPS-induced PDLC injury through regulating the miR-769-5p/HIF3A axis. (a) The expression of HIF3A was detected by qRT-PCR in gingival tissues of patients with CP and healthy controls. ${ }^{* *} P<0.01$ vs. healthy controls. (b) The expression of HIF3A was detected by western blot in LPS-treated PDLCs and control cells. ${ }^{* *} P<0.01$ vs. control cells. (c) The expression of HIF3A was detected by western blot in LPS-treated PDLCs transfected with si-NC, si-MALAT1, or si-MALAT1+miR-769-5p inhibitor. ${ }^{* *} P<0.01$ vs. si-NC; ${ }^{\# \#} P<0.01$ vs. si-MALAT1. (d) Cell viability was detected by MTT assay in LPS-treated PDLCs transfected with siNC, si-MALAT1, miR-769-5p inhibitor, or pcDNA-HIF3A. ${ }^{* *} P<0.01$ vs. si-NC; ${ }^{\# \#} P<0.01$ vs. si-MALAT1. (e-g) The concentrations of IL-6, IL- $1 \beta$, and TNF- $\alpha$ were measured by ELISA in supernatants of LPS-treated PDLCs transfected with si-NC, si-MALAT1, miR-769-5p inhibitor, or pcDNA-HIF3A. ${ }^{* *} P<0.01$ vs. si-NC; ${ }^{\# \#} P<0.01$ vs. si-MALAT1. (h) The protein levels of Bax and Bcl- 2 were detected by western blot in LPS-treated PDLCs transfected with si-NC, si-MALAT1, miR-769-5p inhibitor, or pcDNA-HIF3A. ${ }^{* *} P<0.01$ vs. si-NC; ${ }^{\# \#} P<0.01$ vs. si-MALAT1. (i) The protein level of caspase-3 was detected by western blot in LPS-treated PDLCs transfected with si-NC, si-MALAT1, miR-769-5p inhibitor, or pcDNA-HIF3A. ${ }^{* *} P<0.01$ vs. si-NC; ${ }^{\#} P<0.05$ and ${ }^{\# \#} P<0.01$ vs. si-MALAT1.

\section{Discussion}

CP not only seriously affects oral health but also increases the patient's risk of other chronic diseases [30, 31]. Previous researches have pointed that some lncRNAs are associated with pathogenesis of periodontitis, such as lncRNA OIP5AS1 and LINC00687 [32, 33]. In our study, the results revealed that MALAT1 expression was enhanced in gingival tissues of patients with CP and LPS-treated PDLCs. Knockdown of MALAT1 promoted cell viability and inhibited inflammation and apoptosis in LPS-treated PDLCs. Besides,
MALAT1 targeted miR-769-5p and miR-769-5p targeted HIF3A. A further study demonstrated that knockdown of MALAT1 alleviated LPS-induced PDLC injury by regulating the miR-769-5p/HIF3A axis.

Increasing studies showed that MALAT1 plays a pivotal role in LPS-induced inflammation models, such as acute lung injury [34] and ATDC5 cell inflammatory injury [35]. Recently, studies have demonstrated that MALAT1 participates in the development of periodontitis and is upregulated in PDLSCs [12] and inflammatory gingival tissues of CP [13]. In this study, MALAT1 expression was notably increased in 
gingival tissues of patients with CP and LPS-treated PDLCs, suggesting that MALAT1 was related to pathogenesis of CP. Previous studies demonstrated that MALAT1 overexpression promotes inflammatory cytokine production in LPS-treated HGF cells [13]. Similarly, our results suggested that knockdown of MALAT1 inhibited the secretion of inflammatory cytokines in LPS-treated PDLCs. Meanwhile, we found that MALAT1 knockdown enhanced cell viability and inhibited cell apoptosis in LPS-treated PDLCs. All these results suggested that MALAT1 knockdown may inhibit the occurrence and developments of CP in vitro.

Previous studies showed that MALAT1 is involved in CP pathogenesis by sponging miR-125a-3p [11] or miR-20a [13]. In this study, we found that miR-769-5p was a downstream target of MALAT1 and reversely modulated by MALAT1. Numerous studies indicated that miR-769-5p participates in the growth of some cancers and is reduced in non-small-cell lung carcinoma (NSCLC) [36] and retinoblastoma (RB) [37]. Interestingly, Du et al. have pointed that miR-769-5p expression is decreased in LPS-treated PDLCs [19]. Similarly, our results displayed that miR-769-5p expression was reduced in LPS-treated PDLCs and gingival tissues of patients with CP, suggesting that miR-769-5p may be an anti-inflammatory gene in CP. At present, the function of miR-769-5p has been explored in several types of human cancers. For example, miR-769-5p silencing can inhibit cell viability in gastric cancer cells [38]. Silencing of miR-769-5p obviously inhibits cell viability and promotes cell apoptosis in glioma cell lines [39]. However, the function of miR-769-5p is rarely discussed in inflammatory diseases. In this study, our results cleared that miR-769-5p overexpression enhanced cell viability and inhibited apoptosis in LPS-treated PDLCs. Besides, our study revealed that miR-769-5p overexpression could inhibit the secretion of inflammatory cytokines in LPS-treated PDLCs. The above results suggested that miR-769-5p makes a great deal of contributions on inhibiting the development of CP in vitro. Because MALAT1 directly targeted miR-769-5p, we speculated that MALAT1 knockdown may suppress CP progression by targeting miR-769-5p.

HIF3A, a main gene involved in the homeostatic processes, is commonly involved in chronic inflammation [26]. As a transcription factor for many target genes, HIF3A can be regulated by miR-210 in LPS-treated PDLCs [40] or modulated by miR-429 in human endothelial cells [41]. In this study, HIF3A was a target gene of and negatively regulated by miR-769-5p. Previous researches revealed that HIF3A expression is increased in periodontitis [40] and in LPS-treated BV-2 microglial cells [42]. Similar to previous results, we found that HIF3A expression was also increased in gingival tissues of patients with CP and LPS-treated PDLCs, suggesting that HIF3A may be a proinflammatory gene in CP development. In addition, Cuomo et al. have discovered that HIF3A is involved in inflammatory cell infiltration in a murine model of arteriotomy [43]. Meanwhile, HIF3A can regulate the inhibition effect of miR-210 on secretion of inflammatory factors and cell apoptosis in LPS-treated PDLCs [40]. Thus, we speculated that miR-769-5p overexpression may inhibit inflammation and apoptosis by regulating HIF3A in LPStreated PDLCs. Further studies revealed that miR-769-5p inhi- bition and HIF3A overexpression reversed the influence of MALAT1 silencing on cell viability, inflammatory factor secretion, and apoptosis-related protein levels in LPS-treated PDLCs. Because MALAT1 negatively regulated miR-769-5p and miR-769-5p negatively regulated HIF3A, we speculated that MALAT1 knockdown may alleviate LPS-induced PDLC injury by regulating the miR-769-5p/HIF3A axis.

\section{Conclusion}

In conclusion, this research revealed that the expression of MALAT1 was upregulated in gingival tissues of patients with CP and LPS-treated PDLCs. In addition, knockdown of MALAT1 enhanced cell viability and inhibited inflammation and apoptosis in LPS-treated PDLCs through regulating the miR-769-5p/HIF3A axis. This study may provide a new target for the therapy of CP.

\section{Data Availability}

All data can be obtained by contacting the corresponding author.

\section{Conflicts of Interest}

The authors declare that they have no conflicts of interest.

\section{References}

[1] G. Hajishengallis, "Immunomicrobial pathogenesis of periodontitis: keystones, pathobionts, and host response," Trends in Immunology, vol. 35, no. 1, pp. 3-11, 2014.

[2] B. A. Niemiec, "Periodontal disease," Topics in Companion Animal Medicine, vol. 23, no. 2, pp. 72-80, 2008.

[3] P. E. Petersen and H. Ogawa, "Strengthening the prevention of periodontal disease: the WHO approach," Journal of Periodontology, vol. 76, no. 12, pp. 2187-2193, 2005.

[4] E. M. Cardoso, C. Reis, and M. C. Manzanares-Cespedes, "Chronic periodontitis, inflammatory cytokines, and interrelationship with other chronic diseases," Postgraduate Medicine, vol. 130, no. 1, pp. 98-104, 2018.

[5] J. Slots, "Periodontitis: facts, fallacies and the future," Periodontology 2000, vol. 75, no. 1, pp. 7-23, 2017.

[6] P. J. Batista and H. Y. Chang, "Long noncoding RNAs: cellular address codes in development and disease," Cell, vol. 152, no. 6, pp. 1298-1307, 2013.

[7] Y. Zou, C. Li, F. Shu et al., "IncRNA expression signatures in periodontitis revealed by microarray: the potential role of IncRNAs in periodontitis pathogenesis," Journal of cellular biochemistry, vol. 116, no. 4, pp. 640-647, 2015.

[8] H. Zhou, D. Chen, G. Xie, J. Li, J. Tang, and L. Tang, "LncRNA-mediated ceRNA network was identified as a crucial determinant of differential effects in periodontitis and periimplantitis by high-throughput sequencing," Clinical Implant Dentistry and Related Research, vol. 22, no. 3, pp. 424-450, 2020.

[9] S. H. Jin, R. H. Zhou, X. Y. Guan, J. G. Zhou, and J. G. Liu, "Identification of novel key lncRNAs involved in periodontitis by weighted gene co-expression network analysis," Journal of Periodontal Research, vol. 55, no. 1, pp. 96-106, 2020. 
[10] W. Liu, Y. Zheng, B. Chen, T. Ke, and Z. Shi, "LncRNA papillary thyroid carcinoma susceptibility candidate 3 (PTCSC3) regulates the proliferation of human periodontal ligament stem cells and toll-like receptor 4 (TLR4) expression to improve periodontitis," BMC Oral Health, vol. 19, no. 1, p. 108, 2019.

[11] S. Li, X. Liu, H. Li et al., "Integrated analysis of long noncoding RNA-associated competing endogenous RNA network in periodontitis," Journal of Periodontal Research, vol. 53, no. 4, pp. 495-505, 2018.

[12] P. Chen, Y. Huang, Y. Wang, S. Li, H. Chu, and M. Rong, "MALAT1 overexpression promotes the proliferation of human periodontal ligament stem cells by upregulating fibroblast growth factor 2," Experimental and Therapeutic Medicine, vol. 18, no. 3, pp. 1627-1632, 2019.

[13] J. Li, M. Wang, L. Song, X. Wang, W. Lai, and S. Jiang, "LncRNA MALAT1 regulates inflammatory cytokine production in lipopolysaccharide-stimulated human gingival fibroblasts through sponging miR-20a and activating TLR4 pathway," Journal of Periodontal Research, vol. 55, no. 2, pp. 182-190, 2020.

[14] Y. F. Xie, R. Shu, S. Y. Jiang, D. L. Liu, and X. L. Zhang, "Comparison of microRNA profiles of human periodontal diseased and healthy gingival tissues," International Journal of Oral Science, vol. 3, no. 3, pp. 125-134, 2011.

[15] W. Zhou, L. Su, X. Duan et al., "MicroRNA-21 down-regulates inflammation and inhibits periodontitis," Molecular Immunology, vol. 101, pp. 608-614, 2018.

[16] L. Wang, F. Wu, Y. Song et al., "Long noncoding RNA related to periodontitis interacts with miR-182 to upregulate osteogenic differentiation in periodontal mesenchymal stem cells of periodontitis patients," Cell Death \& Disease, vol. 7, no. 8, article e2327, 2016.

[17] Y. Zheng, C. Dong, J. Yang et al., "Exosomal microRNA-155$5 \mathrm{p}$ from PDLSCs regulated Th17/Treg balance by targeting sirtuin-1 in chronic periodontitis," Journal of Cellular Physiology, vol. 234, no. 11, pp. 20662-20674, 2019.

[18] X. M. Chen, Y. Zhao, X. D. Wu et al., "Novel findings from determination of common expressed plasma exosomal microRNAs in patients with psoriatic arthritis, psoriasis vulgaris, rheumatoid arthritis, and gouty arthritis," Discovery Medicine, vol. 28, no. 151, pp. 47-68, 2019.

[19] A. Du, S. Zhao, L. Wan et al., "MicroRNA expression profile of human periodontal ligament cells under the influence of Porphyromonas gingivalis LPS," Journal of Cellular and Molecular Medicine, vol. 20, no. 7, pp. 1329-1338, 2016.

[20] N. Huang, C. Li, W. Sun, J. Wu, and F. Xiao, "Long non-coding RNA TUG1 participates in LPS-induced periodontitis by regulating miR-498/RORA pathway," Oral Diseases, 2020.

[21] C. Zhang, J. Liu, Y. Zhang et al., "LINC01342 promotes the progression of ovarian cancer by absorbing microRNA-30c2-3p to upregulate HIF3A," Journal of Cellular Physiology, vol. 235, no. 4, pp. 3939-3949, 2020.

[22] M. T. Bjerre, S. H. Strand, M. Nørgaard et al., "Aberrant DOCK2, GRASP, HIF3A and PKFP hypermethylation has potential as a prognostic biomarker for prostate cancer," International Journal of Molecular Sciences, vol. 20, no. 5, p. 1173, 2019.

[23] J. Shen, R. Song, Y. Ye, X. Wu, W. H. Chow, and H. Zhao, "_HIF3A_ DNA methylation, obesity and weight gain, and breast cancer risk among Mexican American women," Obesity Research \& Clinical Practice, vol. 14, no. 6, pp. 548-553, 2020.
[24] X. Zhou, X. Guo, M. Chen, C. Xie, and J. Jiang, "HIF-3 $\alpha$ promotes metastatic phenotypes in pancreatic cancer by transcriptional regulation of the RhoC-ROCK1 signaling pathway," Molecular Cancer Research, vol. 16, no. 1, pp. 124134, 2018.

[25] Y. Zhang, Y. Guo, C. Yang et al., "MicroRNA-300 targets hypoxia inducible factor-3 alpha to inhibit tumorigenesis of human non-small cell lung cancer," Neoplasma, vol. 64, no. 4, pp. 554-562, 2017.

[26] J. L. Ebersole, M. J. Novak, L. Orraca et al., "Hypoxia-inducible transcription factors, HIF1A and HIF2A, increase in aging mucosal tissues," Immunology, vol. 154, no. 3, pp. 452-464, 2018.

[27] S. Jia, X. Yang, X. Yang, and F. Zhang, "Retracted article: MicroRNA-210 protects against periodontitis through targeting HIF- $3 \alpha$ and inhibiting p38MAPK/NF- $\kappa$ B pathway," Artif Cells Nanomed Biotechnol, vol. 48, no. 1, pp. 129136, 2020.

[28] H. Chen, Z. Lan, Q. Li, and Y. Li, "Abnormal expression of long noncoding RNA FGD5-AS1 affects the development of periodontitis through regulating miR-142-3p/SOCS6/NF- $\kappa \mathrm{B}$ pathway," Artif Cells Nanomed Biotechnol, vol. 47, no. 1, pp. 2098-2106, 2019.

[29] K. Liu, H. Meng, and J. Hou, "Characterization of the autocrine/paracrine function of vitamin $\mathrm{D}$ in human gingival fibroblasts and periodontal ligament cells," PLoS One, vol. 7, no. 6, article e39878, 2012.

[30] K. Lundberg, N. Wegner, T. Yucel-Lindberg, and P. J. Venables, "Periodontitis in RA-the citrullinated enolase connection," Nature Reviews Rheumatology, vol. 6, no. 12, pp. 727730, 2010.

[31] Y. W. Han and X. Wang, "Mobile microbiome: oral bacteria in extra-oral infections and inflammation," Journal of Dental Research, vol. 92, no. 6, pp. 485-491, 2013.

[32] F. Sanchez-Munoz, G. Martinez-Coronilla, A. G. Leija-Montoya et al., "Periodontitis may modulate long-non coding RNA expression," Archives of Oral Biology, vol. 95, pp. 9599, 2018.

[33] X. Zhang, L. Ren, X. Yan et al., "Identification of immunerelated lncRNAs in periodontitis reveals regulation network of gene-lncRNA-pathway-immunocyte," International Immunopharmacology, vol. 84, p. 106600, 2020.

[34] L. Dai, G. Zhang, Z. Cheng et al., "Knockdown of LncRNA MALAT1 contributes to the suppression of inflammatory responses by up-regulating miR-146a in LPS-induced acute lung injury," Connective Tissue Research, vol. 59, no. 6, pp. 581-592, 2018.

[35] L. Pan, D. Liu, L. Zhao, L. Wang, M. Xin, and X. Li, "Long noncoding RNA MALAT1 alleviates lipopolysaccharide-induced inflammatory injury by upregulating microRNA-19b in murine chondrogenic ATDC5 cells," Journal of Cellular Biochemistry, vol. 119, no. 12, pp. 10165-10175, 2018.

[36] Z. Yang, J. He, P. Gao et al., "miR-769-5p suppressed cell proliferation, migration and invasion by targeting TGFBR1 in non-small cell lung carcinoma," Oncotarget, vol. 8, no. 69, pp. 113558-113570, 2017.

[37] Y. Dong, G. Wan, P. Yan, C. Qian, F. Li, and G. Peng, "Long noncoding RNA LINC00324 promotes retinoblastoma progression by acting as a competing endogenous RNA for microRNA-769-5p, thereby increasing STAT3 expression," Aging, vol. 12 , no. 9, pp. 7729-7746, 2020. 
[38] P. B. Luan, X. Z. Jia, and J. Yao, “miR-769-5p functions as an oncogene by down-regulating RYBP expression in gastric cancer," European Review for Medical and Pharmacological Sciences, vol. 24, no. 12, pp. 6699-6706, 2020.

[39] M. Chang, P. Yan, B. Zhang et al., "MicroRNA-769-5p promotes the growth of glioma cells by targeting lysine methyltransferase 2A," Oncotargets and Therapy, vol. 12, pp. 91779187, 2019.

[40] S. Jia, X. Yang, X. Yang, and F. Zhang, "MicroRNA-210 protects against periodontitis through targeting HIF- $3 \alpha$ and inhibiting p38MAPK/NF- $\kappa$ B pathway," Artif Cells Nanomed Biotechnol, vol. 48, no. 1, pp. 129-136, 2020.

[41] A. Janaszak-Jasiecka, S. Bartoszewska, K. Kochan et al., "miR429 regulates the transition between hypoxia-inducible factor (HIF)1A and HIF3A expression in human endothelial cells," Scientific Reports, vol. 6, article 22775, 2016.

[42] H. Kumar, J. H. Lim, I. S. Kim, and D. K. Choi, "Differential regulation of HIF- $3 \alpha$ in LPS-induced BV-2 microglial cells: comparison and characterization with HIF-1 $\alpha$," Brain Research, vol. 1610, pp. 33-41, 2015.

[43] F. Cuomo, A. Coppola, C. Botti et al., "Pro-inflammatory cytokines activate hypoxia-inducible factor $3 \alpha$ via epigenetic changes in mesenchymal stromal/stem cells," Scientific Reports, vol. 8, no. 1, p. 5842, 2018. 